\title{
RELAJACIÓN Y SENSIBILIDAD A LA ANSIEDAD
}

\section{BLANCA MAS, EVA GÓMEZ ${ }^{1}$, MARGARITA CABAÑERO ${ }^{1}$ y ROSA M. VALIENTE}

\author{
Universidad Nacional de Educación a Distancia \\ ${ }^{1}$ Hospital Provincial de Toledo
}

(Aceptado en enero de 2003)

\begin{abstract}
El presente estudio busca establecer si la sensibilidad a la ansiedad es un predictor de la posterior aparición de dificultades en la relajación, y si es mejor predictor de este aspecto que el propio diagnóstico clínico o que los niveles de STAI. El estudio aborda una importante cuestión: la posible modificabilidad de la sensibilidad a la ansiedad a través del entrenamiento en relajación muscular. Participaron en este trabajo 21 pacientes ambulatorios de la Unidad de Salud Mental de los Hospitales de Toledo y Valladolid, diagnosticados de Trastorno de Pánico (12) y de otros Trastornos de Ansiedad (9), respectivamente. Asimismo, se contó con un grupo control constituido por 10 sujetos pertenecientes al personal administrativo y sanitario del Hospital Provincial de Toledo. La edad media de los participantes fue de 38,71 años (DT=10,31). Los resultados indican que, a) la puntuación obtenida en el ASI no es un predictor de las dificultades aparecidas en el entrenamiento, b) los valores de la ansiedad estado disminuyen en todos los grupos tras el entrenamiento en relajación, y c) el entrenamiento en relajación reduce de forma significativa las puntuaciones del ASI en los dos grupos clínicos.
\end{abstract}

Palabras clave: Relajación, sensibilidad a la ansiedad, entrenamiento en relajación, ansiedad estado, ansiedad rasgo, ansiedad inducida por relajación, pánico inducido por relajación.

\section{Relaxation and Anxiety Sensitivity}

Our study investigated to what extent the anxiety sensitivity is a good predictor of later difficulties in relaxation, and if it is a better predictor of this aspect than the own clinical diagnosis and/or the levels of trait anxiety (STAI). The study discuses the possible modification of the levels of anxiety sensitivity by mean of the training in muscular relaxation. Twenty one ambulatory patients from the Unit of Mental Health of the Hospitals of Toledo and Valladolid participated in the work. A control group consisted in 10 administrative personnel and sanitarian from the Provincial Hospital of Toledo. The mean age of the participants was 38,71 years $(D T=10,31$ ). The results indicate that, a) the punctuation obtained in ASI isn't a predictor of the difficulties appeared in the training, b) the levels of anxiety state diminished in all of the groups after the relaxation training, and c) anxiety sensitivity decreased significantly in the two clinical groups after relaxation training.

Key words: Relaxation, anxiety sensitivity, relaxation training, state anxiety, trait anxiety, Relaxation-Induced Anxiety (RIA), Relaxation-Induced Panic (RIP).

\section{INTRODUCCIÓN}

La relajación es una habilidad esencial para reducir los estados de ansiedad. Jacobson (1938) en sus trabajos pioneros, basados en la investigación controlada de laboratorio, puso de manifiesto cómo la relajación de la musculatura estriada reducía la actividad cerebral. Hay evi- dencia sobre diversas técnicas de relajación desde la antigüedad, y en la actualidad el entrenamiento autógeno (Schultz, 1932) y, en especial, la relajación muscular se han extendido con éxito a la práctica clínica.

Sin embargo, varios autores como Carrington (1977), Heide y Borkovec $(1983,1984)$ y Adler, Craske y Barlow 
(1987) sacaron a la luz un importante handicap del entrenamiento en relajación: en algunas ocasiones origina o exacerba la sensación de ansiedad o provoca ataques de pánico. Este efecto paradójico fue denominado «ansiedad inducida por relajación» (RIA) o "pánico inducido por relajación» ( $R I P)$. Además, este fenómeno puede aparecer con todas las técnicas de relajación [p.ej., biofeedback (Budzynski, Stoyva y Peffer, 1980), relajación progresiva abreviada (Bernstein y Borkovec, 1975), entrenamiento autógeno, en la forma de descargas autógenas (Schultz, 1932) y en meditación (Lazarus, 1976)]. Aunque no existe demasiada información sobre la prevalencia de este problema, en los pacientes clínicos se ha situado alrededor de un 40\% (Amutio, 1998).

El carácter paradójico de este fenómeno se pone de manifiesto al experimentar, algunos pacientes, ansiedad en ausencia de estimulación frontal (Raskin, Johnson y Rodestvedt, 1973). Cohen, Barlow y Blanchard (1985) observaron durante la relajación monotorizada de dos pacientes, como parte de una evaluación fisiológica estándar, que, aunque en sus registros se observaba un decremento en la tasa cardiaca y en el electromiograma (EMG) frontal -indicadores ambos de que los pacientes estaban adquiriendo un estado de relajación-, hubo, al comienzo del pánico, un incremento súbito en las dos medidas. La temperatura de la mano mostró un patrón de respuesta similar aunque menos drástico. El EMG frontal es una medida compleja que recoge no sólo la información de este músculo, sino que detecta, asimismo, la actividad muscular desde la primera vértebra, y refleja las actividades de tragar, respirar, los movimientos de lengua, labios, párpados y globo ocular. Así, niveles bajos en la actividad del EMG frontal reflejan una relajación general en los músculos faciales, una respiración tranquila, ausencia de la actividad de tra- gar y de movimientos oculares (Stoyva y Budzynski, 1993). El verdadero significado de esta paradoja se aprecia si recordamos que, en un principio, se pensó que la relajación de la musculatura facial está íntimamente ligada a una reducción de la actividad mental y está unida a una sensación subjetiva de somnolencia (Jacobson, 1970).

En sus artículos iniciales Heide y Borkovec $(1983,1984)$ propusieron cinco mecanismos descriptivos para el surgimiento de la RIA: miedo a las sensaciones y cogniciones que aparecen durante la relajación, miedo a perder el control, miedo a los síntomas de ansiedad a los que se atiende durante la relajación, aumento de la conciencia de la insatisfacción con uno mismo y experimentación de activación cognitiva negativa no relacionada con la relajación.

El pánico inducido por relajación se diferencia de la ansiedad inducida por relajación por la rapidez con la que aparece. Todavía no sabemos si estos dos fenómenos comparten los mismos mecanismos subyacentes. Adler et al. (1987) han propuesto como mecanismo del pánico inducido por relajación un modelo de condicionamiento interoceptivo: La anticipación del pánico o el aprendizaje del miedo a la posibilidad de otro ataque de pánico. Unido a esto se encuentra la adquisición de una sensibilidad extrema a los síntomas somáticos. Así, en estas personas, la ocurrencia o conciencia de las sensaciones fisiológicas puede evocar el miedo. Es, según estos autores una fobia a las sensaciones físicas. Este modelo sugiere que los pacientes con pánico son sensibles a patrones específicos de respuestas fisiológicas internas más que a un arousal generalizado. La potencia de este modelo se manifiesta porque el pánico puede aparecer incluso en un contexto con expectativas de relajación.

Todo entrenamiento en relajación debería ayudar a desarrollar una mayor 
receptividad, entendida ésta como la habilidad de tolerar, de aceptar experiencias inciertas, poco familiares o paradójicas. Receptividad que se va consolidando a través de los ciclos cognitivos reestructurantes (Smith, 1990). Los procedimientos de relajación requieren una atención focalizada que conllevan un aumento en la conciencia somática. Además, el cambio durante la relajación hacia una dominancia parasimpática, produce el surgimiento de sensaciones muchas veces novedosas y la propia relajación de la musculatura conlleva la aparición de cambios musculares espontáneos (espasmos, tics, agitación) que pueden ser experimentados con aprensión. Asimismo, la activación somática residual puede ser interpretada como ansiedad. Sabemos, además, que las personas ansiosas son más sensibles a estas percepciones, temen centrar su atención en la experiencia interna por lo que esta forma de focalización les resulta aversiva. Los elevados niveles de autoatención de las personas con alta ansiedad generalizada y trastorno de pánico, la preocupación por los síntomas somáticos y la interpretación catastrofista de tales síntomas, podrían subyacer a la RIA (Wells, 1990) en la que, el sentimiento de miedo y amenaza no vienen determinados por acontecimientos externos sino por los propios cambios internos.

Ahora bien, la ansiedad puede surgir también en la fase de tensión del ciclo de tensión-distensión. Así, en personas ansiosas se ha observado un aumento en esta sensación al focalizar su atención en la tensión muscular pero no al hacerlo en la respiración (Waddel, Barlow y O'Brien, 1984). La tensión muscular constituye una parte de la respuesta de alarma del organismo (Selye, 1974) en la que los músculos están preparados para la acción. Eliminar la tensión muscular en personas crónicamente tensas o con un alto grado de tensión muscular puede originar una sensación repentina de inseguridad ante la notable discrepancia entre el temor subjetivo y el tono relajado de los músculos. Lindeman (1987) sugiere que los pacientes ansiosos previenen constantemente la experiencia de relajación. Mantenerse vigilantes y estar alertas es su mejor protección contra la ansiedad. Dejar el control cognitivo y físico no es pues para ellos una tarea fácil. De manera similar, Ley (1988) ha señalado como un factor importante en el desarrollo de la RIA la tendencia a hiperventilar de los pacientes con pánico, tendencia que no corresponde a la baja activación metabólica de la relajación y se convertiría en una señal de alarma capaz de desencadenar la ansiedad.

El miedo a perder el control durante la relajación también ha sido objeto de estudio. Así, se ha encontrado que algunas características de personalidad, como un locus de control interno (Heide y Borkovec, 1983), el miedo a perder el control o el miedo a ponerse ansioso, correlacionan positivamente con el surgimiento de la RIA (Braith, McCullough y Bush, 1988).

La RIA se ha convertido, por tanto, en un obstáculo para el entrenamiento en relajación. El psicólogo clínico conoce bien las interferencias que este problema ocasiona en el desarrollo de la psicoterapia y los abandonos que provoca. Borkovec (1987) señala: «si a una persona relajarse le provoca ansiedad, aprender a relajarse y aprender a usar esta habilidad para combatir la ansiedad diaria se convertirá en una difícil tarea, pero, si no lo logra, con una técnica u otra, su mejoría se entorpecerá». Por todo ello, es crucial encontrar una solución para su aparición. A esto se une que las personas más propensas a desarrollarla serían aquellas aquejadas de ansiedad crónica (Heide y Borkovec, 1984), ansiedad generalizada (Borkovec y Mathews, 1987) y trastorno de pánico (Wells, 1990). 
Como Adler et al. (1987), pensamos que poder observar el surgimiento, o incluso inducir ansiedad o pánico, proporciona la posibilidad de estudiar empíricamente el grado de miedo de los pacientes con trastornos de ansiedad a las señales somáticas.

Por todo ello, nosotros vemos como una variable crítica en el desarrollo de la RIA, la sensibilidad a la ansiedad, que se caracteriza por el miedo a los síntomas de ansiedad, y surge de la creencia de que estas sensaciones tienen consecuencias perjudiciales ya sean somáticas, psicológicas o sociales (Reiss, McNally, 1985; Reiss, 1991), por lo que es, desde un punto de vista conceptual y empírico, diferente de la ansiedad rasgo. La sensibilidad a la ansiedad es un rasgo relativamente estable de personalidad que se encuentra elevado en los trastornos de ansiedad y constituye un factor de riesgo para desarrollar ataques de pánico (Reiss, 1991; Taylor 1995, Sandín, Chorot y McNally 1996). Asimismo, se ha hipotetizado que constituye una consecuencia secundaria de los propios ataques de pánico (Goldstein y Chambless, 1987).

La sensibilidad a la ansiedad se mide a través del Índice Sensibilidad a la Ansiedad (ASI) (Peterson y Reiss, 1992) que está traducido y baremado para población española (Sandín et al., 1996). Es este un cuestionario corto (16 items) con una buena fiabilidad y validez. Esta medida ha entrado ya a formar parte de la batería de pruebas de la evaluación del trastorno de pánico, tanto al inicio del tratamiento como en su evolución (Penava, Otto, Maki y Pollack, 1998), y ha resultado ser una variable importante para determinar el papel de otras variables, como por ejemplo, la medicación durante las exposiciones (Zollner, 1997). Por ser un índice fiable, independiente de otras variables $\mathrm{e}$ importante en la conducta de miedo (Benton y Allen, 1996; Liebman y Allen, 1996), pensamos que puede tener el potencial necesario para evaluar la actuación de las personas en un entrenamiento en relajación. Parte de la solución del problema de la RIA radica en conocer de antemano qué personas pueden desarrollarla para introducir desde el comienzo de los entrenamientos las actuaciones preventivas necesarias (velocidad del entrenamiento, postura, posición de los ojos bien cerrados o semiabiertos, exposición jerarquizada a las sensaciones, etc.) que redundaría en el éxito de los entrenamientos y en beneficio en la terapia. Esperamos que el poder predictor de este índice sea mejor que el que pueda proporcionar el diagnostico clínico u otras medidas de ansiedad como el STAI. Asimismo esperamos que el propio entrenamiento reduzca los valores de este índice, mostrando una nueva visión del potencial del entrenamiento en relajación en la terapia de los trastornos de ansiedad en general y del trastorno de pánico en particular. Por tanto, el presente estudio busca establecer si la sensibilidad a la ansiedad es un predictor de la posterior aparición de dificultades en la relajación y si es mejor predictor de este aspecto que el propio diagnóstico clínico y que los niveles de STAI. El estudio aborda una tercera cuestión: la posible modificabilidad de la sensibilidad a la ansiedad a través del entrenamiento en relajación muscular.

\section{MÉTODO}

\section{Muestra}

Participaron en el presente estudio pacientes ambulatorios de la Unidad de Salud Mental del Hospital Provincial de Toledo con diagnóstico de Trastorno de Pánico, y pacientes de la Unidad de Salud Mental del Hospital Dr. Villacían de Valladolid diagnosticados de otros trastornos de ansiedad, incluyendo Trastorno de Ansiedad Generalizada, Trastorno de Fobia 
Social y Trastorno Obsesivo-Compulsivo (que constituyeron los grupos experimentales). Además, como grupo de control, participaron sujetos pertenecientes al personal administrativo y sanitario del Hospital Provincial de Toledo sin ninguna psicopatología en el momento del estudio. Para establecer los diagnósticos se siguieron los criterios operativos del DSM-IV (APA 1994) en forma de entrevista clínica. Paralelamente al entrenamiento en relajación, todos los sujetos clínicos estaban sometidos a un tratamiento de corte cognitivo-conductual y, en su mayor parte, a un tratamiento farmacológico.

Se establecieron tres grupos: 9 sujetos formaron el grupo de pánico (GP), 10 sujetos integraron el grupo de otros trastormos de ansiedad (GA), excluyendo el trastorno de pánico, y 12 sujetos formaron el grupo control (GC). Todos los sujetos fueron informados de las características del estudio y participaron en él de modo voluntario. En la Tabla 1 se recogen las características sociodemográficas de la muestra.

La media de edad $(38,71$ años) se encuentra equiparada en los tres grupos, aunque en el GP, tiende a ser ligeramente inferior (34,22 años). Respecto a la distribución por sexo, la proporción de mujeres que tomaron parte en el estudio fue muy superior a la de los varones, fenómeno habitual en las poblaciones clínicas de los Trastornos de Ansiedad. No se observaron diferencias estadísticamente significativas intergrupo en esta variable. La mayor parte de los sujetos que tomaron parte en el estudio estaban casados, en mayor proporción en el GA y en el GC,

\section{Instrumentos psicométricos utilizados}

Todos los sujetos cumplimentaron las versiones adaptadas a la población española de los siguientes cuestionarios:

- Inventario de depresión de Beck (BDI) (Beck, Ward, Mendelson, Mock y Erbaugh, 1961): Versión de 21 items, adaptada para la población española por Conde y Franch (1984). Se cumplimentó antes de iniciar el entrenamiento, en la sesión $1^{a} \mathrm{y}$ al finalizar éste, en la sesión $5^{\mathrm{a}}$.

- Inventario de ansiedad estado rasgo de Spielberger (STAI) (Spielberger, Gorsuch y Lushene, 1970), adaptado para población española por TEA. El STAI-Rasgo se cumplimento antes de iniciar el entrenamiento, en la sesión $1^{\mathrm{a}}$, y al finalizar éste, en la sesión $5^{\mathbf{a}}$. El STAI-Estado se rellenaba antes y después de cada una de las cinco sesiones de entrenamiento.

- Anxiety Sensitivity Index (ASI) (Peterson y Reiss, 1992), se empleó

Tabla 1. Datos descriptivos de la muestra total y subgrupos

\begin{tabular}{|c|c|c|c|c|}
\hline & $\begin{array}{l}\text { MUESTRA TOTAL } \\
\qquad(\mathrm{N}=31)\end{array}$ & $\begin{array}{l}\text { GRUPO } \\
\text { CONTROL (GC) } \\
(\mathrm{N}=12)\end{array}$ & $\begin{array}{c}\text { GRUPO } \\
\text { PÁNICO (GP) } \\
(\mathrm{N}=9)\end{array}$ & $\begin{array}{c}\text { GRUPO OTROS } \\
\text { TR. ANSIEDAD (GA) } \\
(\mathrm{N}=10)\end{array}$ \\
\hline EDAD & $\begin{array}{l}X=38,71 \\
D T=10,31\end{array}$ & $\begin{array}{l}X=40,25 \\
D T=9,77\end{array}$ & $\begin{array}{r}X=34,22 \\
D T=9,71\end{array}$ & $\begin{array}{l}X=40,90 \\
D T=11,18\end{array}$ \\
\hline SEXO & $\begin{array}{l}\text { Varones: } 7(22,6 \%) \\
\text { Mujeres: } 24(77,4 \%)\end{array}$ & $\begin{array}{l}\text { Varones: } 3(25,0 \%) \\
\text { Mujeres: } 9(75,0 \%)\end{array}$ & $\begin{array}{l}\text { Varones: } 3(33,3 \%) \\
\text { Mujeres: } 6(66,6 \%)\end{array}$ & $\begin{array}{l}\text { Varones: } 1(10,0 \%) \\
\text { Mujeres: } 9(90,0 \%)\end{array}$ \\
\hline ESTADO CIVIL & $\begin{array}{c}\text { Solteros: } 9(29,0 \%) \\
\text { Casados: } 19(61,3 \%) \\
\text { Otros: } 3(9,7 \%)\end{array}$ & $\begin{array}{l}\text { Solteros: } 4(33,3 \%) \\
\text { Casados: } 8(66,6 \%)\end{array}$ & $\begin{array}{c}\text { Solteros: } 3(33,3 \%) \\
\text { Casados: } 3(33,3 \%) \\
\text { Otros: } 3(33,3 \%)\end{array}$ & $\begin{array}{l}\text { Solteros: } 2(20,0 \%) \\
\text { Casados: } 8(80,0 \%)\end{array}$ \\
\hline
\end{tabular}


la adaptación española (Sandín, Chorot y McNally, 1996). Se cumplimentó antes de iniciar el entrenamiento, en la sesión $1^{\mathrm{a}}$, y al finalizar éste, en la sesión $5^{\mathrm{a}}$.

- Autorregistros, que los sujetos rellenaron inmediatamente después de cada una de las cinco sesiones de relajación y en los que deben registrar su percepción acerca del grado de relajación obtenida (medido mediante una escala de 0 a10), así como los problemas que hayan podido surgir durante la sesión. Estos autorregistros también se entregaron a los sujetos para que los cumplimentasen diariamente, después de la práctica de la relajación muscular progresiva en sus domicilios.

\section{Procedimiento}

Todos los sujetos acudían una vez por semana a una sesión de relajación muscular progresiva durante cinco semanas consecutivas. Los sujetos de los Grupos Control (GC) y Trastorno de Pánico (GP) acudían conjuntamente a la Unidad de Salud Mental de Toledo y los sujetos del Grupo de otros Trastornos de Ansiedad (GA) acudían a la Unidad de Salud Mental de Valladolid. La duración de las sesiones era aproximadamente de 2 horas.

En todos los grupos de entrenamiento se concedió una importancia especial a los aspectos educativos y también se incidió en las experiencias previas con métodos más o menos estandarizados de relajación. Se abordaron las expectativas y, en su caso, temores o preocupaciones de cada participante. Cada sesión se iniciaba con una fase de socialización y puesta en común de lo realizado durante la semana. El objetivo de esta fase era iniciar la relajación en un ambiente ya dis- tendido y hacer partícipes a todos los sujetos de las sensaciones en su mayor parte placenteras que tenían los demás, y de los beneficios que se extraían de la práctica del método de relajación. Antes de iniciarse el entrenamiento propiamente dicho se introducían los temas novedosos y se cumplimentaba el STAIEstado.

Tras la relajación se volvía a cumplimentar, al mismo tiempo que se reflejaban en un autorregistro las sensaciones y dificultades aparecidas en la sesión. Al finalizar se compartían en grupo las experiencias de los participantes y se insistía en la importancia de la práctica diaria, fuera del ámbito hospitalario, al menos una vez al día. Pretendíamos incidir en el grupo como movilizador para la práctica. Como ayuda adicional en la práctica de la relajación intersesiones se entregó a cada sujeto en la primera sesión, un casete con instrucciones grabadas, con un contenido similar al entrenado en grupo. El desarrollo de las sesiones aparece recogido en el Anexo 1.

\section{Diseño}

El diseño empleado en este estudio es factorial mixto, con dos variables independientes (VIs), una por selección, entre grupos (grupo en el que se ha incluido a cada sujeto en función de su diagnóstico) y otra intragrupo (aprendizaje en el entrenamiento en relajación muscular progresiva). Como variables dependientes (VDs) se consideraron la sintomatología depresiva (BDI), la ansiedad estado-rasgo (STAI), la sensibilidad a las manifestaciones somáticas de ansiedad (ASI) y, a nivel cualitativo, la valoración subjetiva del grado de relajación, los problemas principales y la adherencia a la práctica de la misma fuera de las sesiones semanales de grupo. 


\section{RESULTADOS}

Para las comparaciones intragrupo pre y post sesión/entrenamiento, empleamos la prueba de los pares igualados de Wilcoxon, aplicable para dos muestras relacionadas de pequeño tamaño. Para las comparaciones intergrupo (GP, GA y GC), utilizamos la prueba no paramétrica de Kruskal-Wallis, realizando posteriormente comparaciones dos a dos con la corrección de Bonferroni para evitar el exceso de error Tipo I.

En todos los grupos y en todas las sesiones, las puntuaciones percentílicas del STAI-E previas a la sesión de relajación fueron superiores a las medidas tras el entrenamiento. Además, en todos los grupos, la tendencia a lo largo del aprendizaje en relajación fue a disminuir esos niveles de ansiedad estado tanto iniciales, antes de la sesión, como los posteriores a la sesión. Las puntuaciones que aparecen en la Tabla 2 son el promedio de las puntuaciones centílicas de los sujetos, operación no aplicable a puntuaciones de carácter ordinal como son los centiles pero que ilustra bien esa tendencia.

\section{Comparaciones intragrupo}

Los datos referidos a los diferentes grupos pueden observarse en la Tabla 3 . Para los Grupos de Pánico (GP) y de Ansiedad (GA), se obtiene un descenso en todas las puntuaciones post sesión/ tratamiento respecto de las puntuaciones pre sesión/tratamiento. Todas las diferencias son estadísticamente significativas excepto las referidas al STAI-Rasgo. En el Grupo Control (GC), aparecen diferencias estadísticamente significativas en el STAI-Estado pre y post de las sesiones dos a cinco y en el STAI-Rasgo.

En líneas generales en los tres grupos las puntuaciones de todas las variables disminuyen tras la sesión o el entrenamiento completo en relajación. En los

Tabla 2. Medias y desviaciones típicas (entre paréntesis) para los grupos de trastorno de pánico, trastornos de ansiedad y grupo control en cada una de las variables dependientes

\begin{tabular}{|c|c|c|c|c|c|c|c|c|}
\hline \multirow[b]{2}{*}{ STAIR-Pre } & \multicolumn{2}{|c|}{$\begin{array}{c}G P \\
\text { Media (DT) } \\
\mathrm{N}=\mathbf{9}\end{array}$} & \multicolumn{2}{|c|}{$\begin{array}{c}G A \\
\text { Media (DT) } \\
\mathrm{N}=10\end{array}$} & \multicolumn{2}{|c|}{$\begin{array}{c}G C \\
\text { Media (DT) } \\
\mathrm{N}=12\end{array}$} & \multirow{2}{*}{$\frac{\chi^{2}(2)}{19,82}$} & \multirow{2}{*}{$\begin{array}{c}P \\
0,000\end{array}$} \\
\hline & $78,44^{\mathrm{a}}$ & $(19,82)$ & $76,70^{a}$ & $(18,80)$ & $35,17^{b}$ & $(10,33)$ & & \\
\hline STAIR-Pos & $62,78^{a}$ & $(13,98)$ & $75,30^{a}$ & $(19,97)$ & $23,92^{b}$ & $(17,99)$ & 19,17 & 0,000 \\
\hline STAIE-1Pre & $62,89^{\mathrm{a}}$ & $(28,37)$ & $67,30^{a}$ & $(18,71)$ & $31,67^{b}$ & $(20,24)$ & 11,03 & 0,004 \\
\hline STAIE-1Post & 49,67 & $(30,23)$ & 38,00 & $(26,28)$ & 24,92 & $(22,37)$ & 4,63 & 0,099 \\
\hline STAIE-2Pre & $61,00^{\mathrm{ac}}$ & $(26,98)$ & $53,90^{\mathrm{bc}}$ & $(28,07)$ & $30,08^{\mathrm{b}}$ & $(16,91)$ & 7,39 & 0,025 \\
\hline STAIE-2Post & 33,89 & $(31,96)$ & 39,50 & $(26,12)$ & 20,58 & $(18,50)$ & 2,08 & 0,354 \\
\hline STAIE-3Pre & $55,56^{\circ}$ & $(25,30)$ & $54,90^{\mathrm{a}}$ & $(20,29)$ & $26,17^{b}$ & $(21,61)$ & 9,41 & 0,009 \\
\hline STAIE-3Post & 25,44 & $(22,42)$ & 33,80 & $(25,14)$ & 12,17 & $(13,48)$ & 7,95 & 0,019 \\
\hline STAIE-4Pre & $50,78^{a}$ & $(17,80)$ & $50,30^{a}$ & $(19,79)$ & $28,17^{b}$ & $(20,90)$ & 8,51 & 0,014 \\
\hline STAIE-4Post & 18,33 & $(22,51)$ & 27,90 & $(25,48)$ & 16,92 & $(20,07)$ & 1,19 & 0,553 \\
\hline STAIE-5Pre & $48,44^{\mathrm{ab}}$ & $(25,50)$ & $62,40^{\mathrm{b}}$ & $(24,00)$ & $25,92^{a}$ & $(17,61)$ & 9,96 & 0,007 \\
\hline STAIE-5Post & $24,00^{\mathrm{ab}}$ & $(23,34)$ & $36,40^{b}$ & $(30,32)$ & $11,17^{\mathrm{a}}$ & $(12,36)$ & 5,97 & 0,051 \\
\hline BDI-Pre & $19,00^{\mathrm{a}}$ & $(09,72)$ & $14,30^{\mathrm{a}}$ & $(07,86)$ & $3,42^{b}$ & $(02,57)$ & 16,95 & 0,000 \\
\hline BDI-Post & 08,56 & $(07,32)$ & 09,00 & $(07,54)$ & 2,92 & $(02,35)$ & 7,98 & 0,019 \\
\hline ASI-Pre & $36,67^{a}$ & $(13,19)$ & $38,60^{\mathrm{a}}$ & $(12,96)$ & $11,50^{\mathrm{b}}$ & $(05,55)$ & 19,83 & 0,000 \\
\hline ASI-Post & $21,44^{\mathrm{a}}$ & $(07,21)$ & $28,20^{\mathrm{a}}$ & $(09,37)$ & $9,58^{b}$ & $(05,84)$ & 16,96 & 0,000 \\
\hline
\end{tabular}

Nota: $\chi^{2}$ según prueba de Kruskal-Wallis. Las letras en superíndices indican, según la prueba a posteriori de Bonferroni, qué medias son iguales. ${ }^{*} \mathrm{p}<0,05$. GP = Grupo de trastorno de pánico, $\mathrm{GA}=\mathrm{Grupo}$ de otros trastornos de ansiedad, $\mathrm{GC}=$ Grupo control. 
Tabla 3. Diferencias estadísticas según prueba de pares igualados de Wilcoxon para la comparación de las medidas pre y post entrenamiento en relajación en el grupo de pánico

\begin{tabular}{|c|c|c|c|c|c|c|}
\hline & \multicolumn{2}{|c|}{$G P$} & \multicolumn{2}{|c|}{$G A$} & \multicolumn{2}{|c|}{$G C$} \\
\hline & $Z$ & $p$ & $Z$ & $p$ & $Z$ & $p$ \\
\hline ASI post-pre & $-2,6656$ & $0,01^{* *}$ & $-2,2424$ & $0,02^{\star}$ & $-1,2741$ & 0,20 \\
\hline BDI post-pre & $-2,5205$ & $0,01^{\star \star}$ & $-2,7011$ & $0,01 * \star$ & $-0,5096$ & 0,61 \\
\hline STAI-E 1 post-pre & $-2,5471$ & $0,01^{* *}$ & $-2,8031$ & $0,00^{* \star}$ & $-1,4905$ & 0,14 \\
\hline STAI-E 2 post-pre & $-2,6656$ & 0,01 ** & $-2,0386$ & $0,04^{*}$ & $-2,1181$ & $0,03^{*}$ \\
\hline STAI-E 3 post-pre & $-2,5471$ & 0,01 * * & $-2,0896$ & $0,04^{\star}$ & $-3,0594$ & $0,00^{* *}$ \\
\hline STAI-E 4 post-pre & $-2,6656$ & $0,01^{* *}$ & $-2,2934$ & $0,02^{\star}$ & $-3,0594$ & $0,00^{\star *}$ \\
\hline STAI-E 5 post-pre & $-1,9548$ & $0,05^{\star}$ & $-1,9876$ & $0,05^{\star}$ & $-3,0594$ & $0,00^{\star *}$ \\
\hline STAI-R post-pre & $-1,5993$ & 0,11 & $-0,3554$ & 0,72 & $-2,2228$ & $0,03^{\star}$ \\
\hline
\end{tabular}

Nota: ${ }^{*} p<0,01 ;{ }^{*} p<0,05$

dos grupos clínicos disminuyen significativamente las puntuaciones de las escalas de sensibilidad a la ansiedad y depresión. La mayor parte de las mediciones de ansiedad estado también disminuyen de modo significativo tras la sesión de relajación. La puntuación de ansiedad-rasgo disminuye de forma estadísticamente significativa solo en el grupo control y se mantiene en los dos grupos clínicos.

\section{Comparaciones intergrupo}

La Tabla 2 muestra las comparaciones intergrupo en cada una de las mediciones así como entre cuáles aparecen diferencias estadísticamente significativas.

En las puntuaciones del ASI previas al entrenamiento, las de los dos grupos clínicos son significativamente mayores que las del grupo control pero no existen diferencias entre ellos. Considerando las puntuaciones del ASI tras el entrenamiento en relajación, aunque ha habido una reducción en las puntuaciones estos valores, continúan siendo significativamente mayores que los del grupo control.

En el BDI, se obtienen diferencias estadísticamente significativas en las puntuaciones preentrenamiento entre los grupos clínicos y el grupo control, pero no entre los grupos clínicos. En las puntuaciones postest de los grupos, no se observan diferencias estadísticamente significativas.

En los niveles de ansiedad rasgo, las puntuaciones previas al entrenamiento son superiores, a niveles estadísticamente significativos, en el Grupo de Pánico y Grupo de otros Trastornos de Ansiedad.

En los niveles de ansiedad estado previos a la sesión, hay diferencias significativas entre el grupo control y los otros dos grupos excepto en la segunda sesión respecto del grupo de otros Trastornos de ansiedad y en la sesión quinta respecto del grupo de Trastorno de Pánico. En ninguna medición aparecen diferencias entre los dos grupos clínicos.

En niveles de ansiedad estado medidos tras completar la sesión de entrenamiento grupal no aparecen diferencias estadísticamente significativas entre los grupos salvo en la última sesión, en la que el grupo de otros Trastornos de Ansiedad tiene puntuaciones más elevadas que los otros dos grupos.

\section{DISCUSIÓN}

\section{Problemas encontrados durante el entrenamiento en relajación (véase Anexo 2)}

El problema principal de los pacientes con trastorno de pánico lo constituyeron 
los pensamientos intrusivos que interferían con el seguimiento de las instrucciones. Las cogniciones ansiógenas no asociadas a la relajación son una faceta de la RIA, pero nosotros esperábamos, especialmente en estos pacientes, que la RIA se manifestase en forma de sensibilidad a la ansiedad, cosa que tampoco ocurrió en los pacientes con otros trastornos de ansiedad, a pesar de que las puntuaciones de ambos grupos en el ASI eran muy altas.

El resto de los problemas recogidos no pueden considerarse manifestaciones de ansiedad. Ninguna de las personas sintió miedo ante las tensiones musculares ni ante las tensiones residuales que en algunos casos son evaluadas como dolor. Estas tensiones suelen ser normales cuando el entrenamiento se realiza sentado en una silla y no tumbado o en un sillón de relajación. La cara suele ser, para las personas tensas, una de las zonas más difíciles de relajar y requieren un tiempo para aprender a relajarse completamente, como de hecho, ocurrió.

El picor generalizado puede ser debido a la vasodilatación parasimpática, pero el paciente con trastorno de pánico no la evalúa con miedo.

Aunque hubo mareos, producidos bien por la hora en la que se realizaba el entrenamiento (16 horas, por lo que algunas personas podían estar haciendo la digestión), bien por el cambio en el patrón respiratorio, ninguna persona tuvo miedo a esta sensación (ítem n. ${ }^{\circ} 4$ del ASI) que desaparecía al abrir los ojos. Además este ítem era el que puntuaba como más elevado en el ASI previo, pero el que apareciera la sensación de mareo durante la relajación, no produjo emoción negativa.

No es fácil catalogar el miedo a cerrar los ojos, pero por el momento en el que aparecio, nos inclinamos a pensar que representa más el miedo a perder el control que un miedo a las sensaciones corporales.
Por último, aunque quedarse dormido es un problema para el buen aprendizaje de la técnica, no denota la existencia de una actividad fisiologica o cognitiva alta.

La propia sensación de estar relajado fue valorada por todos los participantes como positiva y no como aversiva o desagradable.

En este trabajo, los únicos pacientes que mostraron problemas relacionados con la RIA, fueron los diagnosticados con trastorno de pánico, pero todos estos problemas estaban relacionados con cogniciones negativas intrusivas y no con el entrenamiento. El ASI no se ha mostrado en este trabajo como un adecuado predictor del curso del entrenamiento, ni han aparecido miedos relacionados con las sensaciones novedosas extrañas de la relajación ni con las tensiones musculares.

Estos datos son prometedores para conocer mejor el fenómeno de la RIA y los factores involucrados en su desarrollo. Consideramos necesaria mayor investigación específica que permita aseverar que durante el entrenamiento en relajación los problemas relacionados con la misma aparecen bajo la forma de cogniciones inadecuadas y miedo a perder el control. Estos trabajos deberían extenderse a otras técnicas de relajación ante la posibilidad de que cada tipo de entrenamiento conlleve unos problemas específicos.

\section{Sensibilidad a la Ansiedad}

El estudio se diseñó siguiendo los planteamientos teóricos del constructo de sensibilidad a la ansiedad. En contra de lo esperable según los estudios de validación española (Sandín, Chorot y McNally, 1996), aunque las puntuaciones de los grupos clínicos con psicopatología de ansiedad, son puntuaciones más elevadas que las del grupo control, no difieren entre ellos significativamente.

En nuestro trabajo, el ASI, no permite discriminar el trastorno de pánico de los 
otros trastornos de ansiedad. Este resultado puede ser debido al reducido número de la muestra de estudio, pero puede ser también debido a que nuestros pacientes cumplimentaron el ASI antes de haber recibido ningún tipo de tratamiento. La terapia cognitiva (Penava et al.,1998) así como el entrenamiento en relajación (ver muestras de datos), reducen los valores del ASI. Como los datos obtenidos en el estudio de Canarias, son en todos los grupos inferiores a las muestras, cabía esperar ciertas diferencias entre los habitantes de las islas y los de la Península, aunque esto no explicaría la similitud de las puntuaciones de los grupos clínicos. Antes de sacar conclusiones precipitadas y dado que éste no era el objetivo del presente trabajo, creemos que se necesita más información sobre la distribución de los grupos patológicos en los valores del ASI, recogiendo estos datos antes de comenzar ningún tipo de intervención terapéutica.

Es también notable señalar cómo las puntuaciones del ASI se reducen significativamente en los dos grupos clínicos. También se reduce este índice en el grupo no clínico, pero por provenir de niveles ya bajos, esta diferencia no es significativa.

En estado de relajación se reduce, pues, la aprensión o el miedo con el que se evalúan las sensaciones corporales. Si existiera un índice contrario, tal vez nos permitiría comprobar que, además, paulatinamente las sensaciones corporales se evalúan con adjetivos más benéficos.

El cambio en los valores del ASI no es uniforme (véase Anexo 3), encontrando una reducción más marcada en los items n. ${ }^{9} 4,16$ y 9: «me asusto cuando siento que me desmayo o mareo", "me asusto cuando estoy nervioso», y "cuando noto que mi corazón está latiendo muy deprisa me preocupa que pueda estar teniendo un ataque cardiaco", respectivamente. En estudios futuros sería relevante analizar si estos items son los más discri- minativos y los más sensibles al cambio en los pacientes con trastorno de pánico. Del mismo modo que poder compararlos con el resto de los trastornos de ansiedad: en nuestro estudio, vemos que en grupo de Ansiedad, los items que más se reducen con el entrenamiento en relajación, son el n. ${ }^{2} 14$ ( «las sensaciones corporales poco habituales (raras) me asustan»), el 15 ("cuando estoy nervioso/a me preocupa que pueda estar mentalmente enfermo/a»), y el 5 ("es importante para mí mantener el control de mis emociones»).

De forma general, estos datos nos muestran el beneficio específico del entrenamiento en relajación en los trastornos de ansiedad en general y de pánico en particular y aportan evidencia colateral sobre el desarrollo de la habilidad cognitiva de receptividad propuesta por Smith. Dado que este autor ha desarrollado el cuestionario "las escalas provisionales de estado-rasgo de las dimensiones cognitivas de la relajación" (Smith, 1990), para investigar el cambio cognitivo en la relajación, pensamos que podría emplearse en estudios posteriores junto con el ASI. Sería interesante comprobar si las medidas del ASI, correlacionan negativamente con receptividad y si es el fortalecimiento de esta habilidad cognitiva el responsable de los cambios observados en nuestro trabajo con el ASI.

\section{REFERENCIAS BIBLIOGRÁFICAS}

Adler, C.M., Craske, M.G., y Barlow, D.H. (1987). Relaxation-Induced Panic (RIP): When resting isn't peaceful. Integrated Psychiatry, 5, 94-112.

American Psychiatric Association (APA) (1994). Diagnostic and statistical manual of mental disorders ( $4^{\text {th }}$ ed.). Washington, DC: Autor.

Amutio, A. (1998). Nuevas perspectivas sobre la relajación. Bilbao: Desclée de Brouwer. Beck, A.T., Ward, C.H., Mendelshon, M., Mock, J., y Erbaugh, J. (1961). An inventory 
for measuring depression. Archives of General Psychiatry, 4, 561-571.

Benton, F.A., y Allen, G.J. (1996). Relationships between anxiety sensitivity, emotional arousal, and interpersonal distress. Journal of Anxiety Disorders, 10, 267-282.

Bernstein, D.A., y Borkovec, T.D. (1973). Progressive relaxation training: $A$ manual for the helping professions. Champaing, III: Research Press. (Versión en castellano: Entrenamiento en relajación progresiva, 1975. Bilbao: Desclée de Brouwer).

Borkovec, T.D. (1987). Relaxation-Induced Panic. Commentary. Integrated Psychiatry, 5, 104-106.

Borkovec, T.D., y Mathews, A.M. (1987). The effects of relaxation training with cognitive or nondirective therapy and the role of Relaxation-Induced Anxiety in the treatment of Generalized Anxiety. Journal or Consulting and Clinical Psychology, 55, 883-888.

Braith, J.A., McCullough, J.P., y Bush, J.P. (1988). Relaxation Induced Anxiety in a subclinical sample of chronically anxious subjects. Journal of Behavior Therapy and Experimental Psychiatry, 19, 193-198.

Budzynski, T.H., Stoyva, J.M., y Peffer, K.E. (1980). Biofeedback techniques in psychosomatic disorders. En A. Goldstein y E.B. Foa (Eds.), Handbook of Behavioral Interventions. Nueva York: Wiley.

Carrington, P. (1977). Freedom in Meditation. Nueva York: Doubleday-Anchor.

Cohen, A., Barlow, D., y Blanchard, E. (1985). The psychophysiology of relaxation-associated panic attacks. Journal of Abnormal Psychology, 94, 96-101.

Conde, V., y Franch, J.I. (1984). Escalas de evaluación comportamental para la cuantificación de la sintomatología psicopatológica en los trastornos angustiosos $y$ depresivos. Valencia: Upjohn Farmacoquímica.

Goldstein, A.J., y Chambless, D.L. (1987). A reanalysis of agoraphobia. Behavior Therapy, 9, 47-59.

Heide, F.J., y Borkovec, T.D. (1983). Relaxation-induced anxiety: Paradoxical anxiety enhancement due to relaxation training. Journal of Consulting Clinical Psychology, 51, 171-182.
Heide, F.J., y Borkovec, T.D. (1984). Relaxation-induced anxiety: Mechanism and Theoretical implications. Behavior Research and Therapy, 22, 1-12.

Jacobson, E. (1938). Progressive Relaxation. University of Chicago Press.

Jacobson, E. (1970). Modern treatment of tense patients. Springfield, IL: Charles C. Thomas.

Lazarus, A.A. (1976). Psychiatric problems precipitated by transcendental meditation. Psychological Report, 39, 601-602.

Ley, R. (1988). Panic attacks during relaxation and relaxation-induced anxiety: a hyperventilation interpretation. Journal of Behavior Therapy Experimental Psychiatry, 19, 253-259.

Liebman, S.E., y Allen, G.J. (1996). Anxiety sensitivity, state anxiety and perceptions of facial emotions. Journal of Anxiety Disorders, 9, 257-267.

Lindeman, C.L. (1987). Relaxation-Induced Panic. Commentary. Integrated Psychiatry, 5, 109-110.

Penava, S.J., Otto, M.W., Maki, K.M., y Pollack, M.H. (1998). Rate of improvement during cognitive-behavioral group treatment for panic disorders. Behavior Research and Therapy, 36, 665-673.

Peterson, R.A., y Reiss, S. (1992). Anxiety Sensitivity Index Manual (2nd Edition). Worthington, OH: International Diagnostic Systems.

Raskin, M., Johnson, G., y Rondestvedt, J.W. (1973). Chronic anxiety treated by feedback-induced muscle relaxation. Archives of General Psychiatry, 28, 263-267.

Reiss, S. (1991). Expectancy model of fear, anxiety and panic. Clinical Psychological Review, 11, 141-153.

Reiss, S., y McNally, R.J. (1985). Expectancy model of fear. En S. Reiss y R.R. Bootzin (Ed.), Theoretical Issues in Behavior Therapy (pp. 107-122). Nueva York: Academic Press.

Sandín, B., Chorot, P. y McNally, R.J. (1996). Validation of the Spanish version of the Anxiety Sensitivity Index in a clinical sample. Behavior Research and Therapy, 34, 283-290.

Selye, H. (1974). Stress without distress. Londres: Hodder \& Stoughton. 
Schultz, J.H. (1932). Das Autogene TrainingKonzentrative Selbstentspannung. Leipzig: Thieme.

Smith, J.C. (1990). Cognitive-behavioral relaxation training: $A$ new system of strategies for treatment and assessment. New York: Springer.

Spielberger, C.D., Gorsuch, R.L., y Lushene, R.E. (1970). Manual for the State Trait Anxiety Inventory. Palo Alto, California: Consulting Psychological Press.

Stoyva, J.M., y Budzynski, T.H. (1993). Biofeedback Methods in the Treatment of Anxiety and Stress Disorder. New York: Guilford.

Taylor, S.E. (1995). Health psychology. New York: McGraw-Hill.
Waddel, M.T., Barlow, D.H., y O'Brien, G.T. (1984). A preliminary investigation of cognitive and relaxation treatment of panic disorder: Effects on intense anxiety vs. «background" anxiety. Behavior Research and Therapy, 22, 393-402.

Wells, A. (1990). Panic Disorder in Association with Relaxation Induced Anxiety: an attentional training approach to treatment. Behavior Therapy, 21, 273-280.

Zollner, L. (1997). Contextual effects of alprazolam on exposure therapy. Dissertation Abstracts International: Section $B$ : The Sciences \& Engineering, 57 (11-B), 7242.

ANEXO 1. Desarrollo detallado de cada sesión del entrenamiento en Relajación Muscular Progresiva

En nuestro trabajo hemos realizado un entrenamiento en relajación muscular con algunas características propias: en la primera fase, los grupos musculares entrenados eran los mismos que los propuestos por Berstein y Borkovec (1975), pero su aprendizaje se dividió en dos sesiones (el primer día se entrenó sólo hasta la cara) y además se introdujo desde el principio un entrenamiento en respiración abdominal, lenta, profunda, especialmente indicada en los trastornos en los que la hiperventilación es un problema falta referencia.

En la segunda fase, aunque se reducen los grupos musculares, se reduce también la tensión de cada grupo. La tercera fase es una profundización en las sensaciones de relajación con sólo tensión general al comienzo. A partir de la fase segunda cada sesión terminaba con una visualización relajante. Se introdujo, por último, una palabra clave de relajación. En todo momento se evita la sugestión y la hipnosis. Este entrenamiento es efectivo para producir una clara sensación de calma. Las potencialidades de este entrenamiento, que pasamos a detallar, merecen ser estudiadás detenidamente.

\section{PRIMERA SESIÓN:}

- Presentación

- Expectativas, creencias, conocimientos previos

- Motivación

- Cuestionarios: STAI, BDI, ASI.

- Introducción a la técnica

- Breves explicaciones del concepto de estrés

- Entrenamiento en respiración diafragmática

- Instrucciones específicas de tensión de cada grupo muscular

- Entrenamiento en tensar/relajar los siguientes grupos musculares

- Relajación: Fase 1. Relajación hasta la cara

- Entrenamiento en cumplimentar autorregistros. STAI-E

- Trabajo para casa

SEGUNDA SESIÓN:

- Revisión del trabajo en casa 
- STAI-E

- Se confeccionan las imágenes relajantes

- Fase I completa: Entrenamiento muscular completo

- Introducción palabra clave al espirar (relax, calma, paz)

- Autorregistros y STAI-E

- Tarea para casa

TERCERA SESIÓN:

- Revisión del trabajo en casa

- STAI-E

- Imágenes relajantes

- Fase 2 del entrenamiento: Entrenamiento muscular con tensión ligera/relajación de 7 grupos musculares

- Detección de tensión y relajación en los grupos musculares anteriores

- Imágenes relajantes

- Autorregistros y STAI-E

- Tarea para casa

\section{CUARTA SESIÓN}

- Revisión trabajo en casa

- STAI-E

- Imágenes relajantes

- Repetición de la fase dos del entrenamiento

- Autorregistros y STAI-E

- Tarea para casa

QUINTA SESIÓN:

- Revisión trabajo en casa

- STAI-E

- Fase 3 de relajación:

- Tensión simultanea de los músculos entrenados

- Detección de tensión

- Profundización en la relajación y sus sensaciones

- Respiración (con palabra clave)

- Imágenes relajantes

- Autonomía para la relajación

- Autorregistros

- Cuestionarios: STAI, BDI, ASI.

- Tarea para casa

- Despedida

ANEXO 2. Listado de problemas aparecidos durante el entrenamiento en relajación (Tabla I)

1. En tres sujetos aparece un ligero mareo, que suele desaparecer al abrir los ojos. Dos sujetos con pánico y uno con otros trastornos de ansiedad. Los sujetos con pánico sometidos a entrenamiento vivían esta sensación sin malestar y desaparecía al hacer la relajación con los ojos abiertos o abrirlos esporádicamente.

2. Dos sujetos se quedaron dormidos durante la $3^{\mathrm{a}}$ sesión en grupo. Este problema aparece más frecuentemente en la práctica diaria en casa. (Grupo control exclusivamente). 
3. En las tres primeras sesiones, aparece dificultad en más de la mitad de los sujetos en relajar los músculos de la cara. Este problema va desapareciendo progresivamente con la práctica diaria, reduciéndose al mínimo en las dos últimas sesiones. (En todos los grupos, aunque tardan más en desaparecer en los sujetos con pánico).

4. Sólo en un sujeto aparece tensión en el cuello y espalda al finalizar los ejercicios de relajación (un sujeto del grupo de pánico). Este problema se solucionó indicándole que tensase menos estas zonas. Los ejercicios de relajación se hacían en sillas bastante duras.

5. Un problema bastante generalizado en el grupo fue el de coordinar la respiración lenta con los ejercicios de tensión-relajación. Desaparece con el entrenamiento diario. Más difícil de solucionar en sujetos con pánico y con otros trastornos de ansiedad que en los del grupo control.

6. Falta de concentración en las instrucciones. Pensamientos ansiogenos intrusivos. Problema principal en algunos (6) pacientes del grupo de pánico. Los pensamientos que aparecen principalmente son el miedo a perder el control y el no conseguir relajarse y, por lo tanto, a no estar haciéndolo bien. También aparece en el grupo de otros trastornos de ansiedad (4), aunque en menor medida.

7. En un paciente con trastorno de pánico, se produjo un efecto paradójico de la relajación: «si cierro los ojos, siento que me voy a morir«. Preocupación por conseguir un estado de relajación que interfiere el objetivo. En este sujeto el trastorno de pánico estaba muy cronificado, de larga evolución. Esto sucede en las sesión n. ${ }^{2} 1$, al comienzo del entrenamiento. Fue abordado de forma individual.

8. Tensión en el cuello y hombros incluso al relajarlos, referido por ocho sujetos. Estos sujetos eran tanto de pánico como de otros trastornos de ansiedad.

9. En un sujeto, picor en todo el cuerpo al comienzo del entrenamiento. Del grupo de pánico, mujer y con unos rasgos de personalidad histriónicos.

10. Dificultades en visualizar escena relajante. Problema muy generalizado sin diferencia significativas entre los tres grupos.

Casi todos los sujetos consiguen un grado de relajación entre 6-9 desde las primeras sesiones de entrenamiento, medido con una escala subjetiva de 0-10.

Las sensaciones de relajación más frecuentes (véase Tabla II), percibidas como muy agradables fueron: peso, calor, sueño, estado de descanso. Estas sensaciones y el grado de relajación obtenido fueron mejores o más intensos durante el entrenamiento semanal en grupo que en la práctica individual diaria.

Tabla I. Listado de problemas según porcentajes de aparición en cada sujeto al menos en un momento del entrenamiento

\begin{tabular}{lccc}
\hline PROBLEMA & GRUPO CONTROL & GRUPO PANICO & GRUPO ANSIEDAD \\
\hline Mareo & 0 & 22,22 & 10 \\
Fuerte somnolencia & 25 & 10,00 & 0 \\
Dificultad relajar músculos de la cara & 50 & 66,67 & 60 \\
Tensión residual en cuello y espalda & 10 & 22,22 & 20 \\
Pensamientos ansiógenos & 0 & 33,33 & 30 \\
Dificultad visualizar imagen relajante & 20 & 22,22 & 40 \\
Efecto paradójico de relajación & 0 & 10,00 & 0 \\
\hline
\end{tabular}


Tabla II. Porcentaje de sujetos que al menos en una ocasión refirieron cada una de estas sensaciones

\begin{tabular}{lrrr}
\hline SENSACION & GRUPO CONTROL & GRUPO PANICO & GRUPO ANSIEDAD \\
\hline Calor & 100,00 & 100,00 & 100 \\
Pesadez & 25,00 & 22,22 & 30 \\
Falta de peso, ligereza & 58,33 & 44,44 & 60 \\
Hormigueo & 16,00 & 33,33 & 20 \\
Bienestar, buen humor & 100,00 & 88,88 & 100 \\
\hline
\end{tabular}

ANEXO 3. Análisis cualitativo de los items del ASI en el grupo de trastornos de pánico (Tablas III y IV)

Destaca el descenso en las puntuaciones de todos los items. El ítem que alcanza una puntuación media más alta en el pretest del grupo de Pánico es el $n .^{9} 4:$ :me asusto cuando siento que me desmayo o me mareo", seguido del f́tem n. ${ }^{9} 16$ : "me asusto cuando estoy nervioso". La disminución de las puntuaciones en estos items en el postest, al finalizar el entrenamiento en relajación, es la más relevante, seguidas de los items $n .{ }^{9} 6$ : "me asusto cuando mi corazón late de forma rápida «, $n .^{\circledR} 14$ : «las sensaciones corporales poco habituales (raras) me asustan», y el ítem n.. 10: «me asusto cuando no puedo respirar hondo o respiro con dificultad». Los items 4 y 16, son los que presentan una reducción más significativa tras el entrenamiento. Esto nos hace pensar que son los items más relevantes y también los más sensibles al entrenamiento en relajación. Los menos sensibles a la experiencia de la relajación son el f́tem n. ${ }^{\circ}$ 1: "para mí es importante no dar la impresión de estar nervioso ", el ítem n. ${ }^{\circledR}$ 7: «me resulta embarazoso o molesto cuando mi corazón hace ruidos", y el ítem n. ${ }^{2}$ 13: "cuando tiemblo la gente suele darse cuenta». Es interesante comprobar que los items con un claro componente cognitivo, son más resistentes al cambio y quizás precisen mayor número de sesiones de entrenamiento para apreciar alguna modificación.

En el grupo de otros trastornos de ansiedad, la mayor intensidad pre-entrenamiento la presentan los items n..$^{\circledR}$ 5: "es importante para mí mantener el control de mis emociones», n. ${ }^{2} 14: » l a s$ sensaciones corporales poco habituales (raras), me asustan", y el ítem $n .^{\circ} 10:$ me asusto cuando no puedo respirar hondo o respiro con dificultad». En el primer ítem se aprecia un claro componente cognitivo que sería coherente con otros trastornos de ansiedad diferentes al pánico, puesto que en dichos trastornos no es relevante la sensibilidad a la ansiedad como componente central del trastorno. En cambio los dos items restantes tienen un componente claro referido a sensaciones corporales.

Son resistentes al cambio los items $n .^{\circledR} 1$ : "para mí es importante no dar la sensación de estar nervioso» y el ítem $n .{ }^{\circ} 4$ : «me asusto cuando siento que me desmayo o mareo «.

Para una visión global de estos resultados, se adjunta una tabla de las puntuaciones medias en cada ítem pre y postest y su gráfica correspondiente.

En futuros estudios sería relevante analizar si estos items son los más discriminativos y los más sensibles al cambio en pacientes con Trastorno de Pánico.

Tabla III. Análisis de los items del ASI en Grupo de Pánico

\begin{tabular}{rccc}
\hline ITEM & MEDIAS PRE & MEDIAS POST & DIF \\
\hline 4 & 3,63 & 2,00 & 1,63 \\
16 & 3,00 & 1,38 & 1,62 \\
9 & 2,75 & 1,29 & 1,46 \\
6 & 2,88 & 1,50 & 1,38 \\
12 & 1,88 & 0,63 & 1,25 \\
14 & 2,63 & 1,38 & 1,25 \\
\hline
\end{tabular}


Tabla III. Análisis de los items del ASI en Grupo de Pánico (Cont.)

\begin{tabular}{rccc}
\hline ÍTEM & MEDIAS PRE & MEDIAS POST & DIF \\
\hline 10 & 2,50 & 1,38 & 1,12 \\
2 & 1,38 & 0,50 & 0,88 \\
3 & 2,38 & 1,50 & 0,88 \\
8 & 2,88 & 2,00 & 0,88 \\
15 & 1,75 & 0,88 & 0,87 \\
11 & 1,13 & 0,50 & 0,63 \\
1 & 2,75 & 2,25 & 0,50 \\
7 & 1,13 & 0,63 & 0,50 \\
13 & 1,25 & 0,75 & 0,50 \\
5 & 2,88 & 2,63 & 0,25 \\
\hline
\end{tabular}

Tabla IV. Análisis de los items del ASI en Grupo de Ansiedad

\begin{tabular}{rccc}
\hline ÍTEM & MEDIAS PRE & MEDIAS POST & DIFERENCIA \\
\hline 14 & 3,1 & 1,8 & 1,3 \\
15 & 2,4 & 1,3 & 1,1 \\
5 & 3,5 & 2,5 & 1,0 \\
8 & 2,7 & 1,7 & 1,0 \\
11 & 2,1 & 1,1 & 1,0 \\
12 & 2,6 & 1,7 & 0,9 \\
2 & 1,8 & 1,0 & 0,8 \\
3 & 2,7 & 1,9 & 0,8 \\
9 & 2,3 & 1,5 & 0,8 \\
13 & 1,8 & 1,1 & 0,7 \\
6 & 2,7 & 2,1 & 0,6 \\
10 & 2,8 & 2,4 & 0,4 \\
7 & 1,6 & 1,4 & 0,2 \\
16 & 2,2 & 2,0 & 0,2 \\
1 & 1,6 & 1,7 & $-0,1$ \\
4 & 2,6 & 2,7 & $-0,1$ \\
\hline
\end{tabular}

